\title{
Predicción del compromiso en Educación Física desde la teoría de la autodeterminación: análisis de invarianza según el nivel de actividad física
}

\author{
Prediction of engagement in physical education through self-determination theory: \\ Invariance across different physical activity levels
}

\author{
Javier Coterón ${ }^{1}$, Evelia Franco ${ }^{2}$, Alberto Almena ${ }^{2}$ \\ 1 Facultad de Ciencias de la Actividad Física y del Deporte-INEF. Universidad Politécnica de Madrid. España. \\ 2 Facultad de Ciencias Humanas y Sociales. Universidad Pontificia Comillas. España.
}

\author{
CORRESPONDENCIA: \\ Evelia Franco Álvarez \\ efalvarez@comillas.edu
}

Recepción: febrero 2020 • Aceptación: julio 2020

\section{CÓMO CITAR EL ARTÍCULO:}

Coterón, J., Franco, E., \& Almena, A. (2020). Predicción del compromiso en Educación Física desde la teoría de la autodeterminación: análisis de invarianza según el nivel de actividad física. Cultura, Ciencia y Deporte, 15(46), 485-494.

\section{Resumen}

El objetivo principal de este trabajo fue testar un modelo predictivo del compromiso comportamental en educación física (EF) desde los postulados de la teoría de la autodeterminación (necesidades psicológicas básicas $\rightarrow$ motivación intrínseca $\rightarrow$ compromiso comportamental) y analizar la estabilidad de dicho modelo en función del nivel de práctica de actividad física (AF). Una muestra de 468 estudiantes de educación física (EF) de entre 12 y 16 años respondieron diferentes cuestionarios validados. La secuencia de relaciones hipotetizada se testó a través de un modelo de ecuaciones estructurales. Los índices de ajuste indicaron que dicho modelo resultó adecuado para predecir el compromiso ( $\chi 2$ [112] = $310.65, \mathrm{p}<.001, \chi 2 / \mathrm{df}=2.77, \mathrm{CFI}=.94, \mathrm{TLI}=.92, \mathrm{RMSEA}$ $=.06$, SRMR $=.06)$. Todas las relaciones hipotetizadas resultaron significativas a excepción de la satisfacción de la necesidad de relación sobre la motivación intrínseca $(p>.05)$. Los hallazgos del análisis de invarianza señalaron que la satisfacción de competencia cobró más importancia entre los adolescentes con mayor nivel de $A F$, siendo la satisfacción de autonomía más relevante en los estudiantes de nivel bajo de AF. Los resultados sugieren que las estrategias utilizadas para favorecer el compromiso en las clases de EF deberían adaptarse a los alumnos en función de la práctica de AF que realicen.

Palabras clave: compromiso comportamental, motivación, educación física, necesidades psicológicas básicas

\begin{abstract}
This study aimed to test a predictive model for behavioral engagement in physical education $(\mathrm{PE})$ in the light of self-determination postulates (basic psychological needs $\rightarrow$ intrinsic motivation $\rightarrow$ behavioral engagement) as well as to analyze the invariance of this model according to physical activity (PA) levels. A sample of 468 12-16 physical education (PE) students completed different validated instruments. The hypothesized sequence was tested through a structural equation model. Fitting indices revealed that the model was suitable to predict engagement $\left(\chi^{2}[112]=310.65, p<.001, \chi^{2} / \mathrm{df}=2.77\right.$, $\mathrm{CFI}=.94, \mathrm{TLI}=.92, \mathrm{RMSEA}=.06, \mathrm{SRMR}=.06$ ). All the hypothesized relationships were significant except for the association between relatedness and intrinsic motivation ( $p>05$ ). Findings from the invariance analyses indicated that competence became more important among adolescents showing higher levels in autonomous motivation and PA while autonomy became more relevant among students showing lower levels in autonomous motivation and PA. Results thus suggest that strategies used to facilitate engagement in PE classes should be adapted to students according to the PA they usually perform.
\end{abstract}

Key words: behavioral engagement, motivation, physical education, basic psychological needs 


\section{Introducción}

Uno de los principales objetivos de la Educación Física (EF) es transmitir a los estudiantes la importancia de la actividad física (AF) y promover la adopción de hábitos responsables de AF regular (Molina et al., 2016). En esta línea, el contexto de la clase de EF ha sido señalado durante las últimas décadas como un lugar muy relevante para favorecer la adherencia a un estilo de vida activo (Albarracín et al., 2014). Sin embargo, si no se establecen condiciones adecuadas, puede generar en el alumnado experiencias negativas que afecten directamente a su nivel de práctica de $\mathrm{AF}$ (Beltrán-Carrillo \& Devís-Devís, 2019) o lo distancien con el tiempo de la práctica regular (Ladwig et al., 2018). En este contexto, promover el compromiso de los estudiantes se ha convertido en un aspecto clave para educadores e investigadores (Franco et al. 2019).

El compromiso de los estudiantes es un constructo multidimensional que incluye aspectos comportamentales, emocionales y cognitivos (Fredricks et al., 2004). La mayor parte de trabajos que han abordado el estudio del compromiso en EF se han centrado en el componente comportamental, que incluye aspectos tales como participar activamente en la clase y escuchar con atención al profesor (Hospel et al., 2016), existiendo evidencia de que este compromiso comportamental predice tanto el rendimiento como el aprendizaje de los alumnos (Skinner et al., 2008). En el caso de la clase de EF una mejora en el aprendizaje de las habilidades motoras adquiere una especial importancia, ya que dicha mejora puede facilitar el éxito y la adherencia en actividades físicas futuras, contribuyendo de este modo al objetivo señalado anteriormente de que los alumnos adopten hábitos responsables de AF regular.

Existe evidencia de que los procesos motivacionales de los alumnos en relación a la EF van a afectar a su compromiso en esta asignatura y la teoría de la autodeterminación (TAD; Ryan \& Deci, 2020) ofrece un enfoque para explicar los mecanismos de dicha asociación entre motivación y compromiso de los estudiantes en EF (Cuevas et al., 2018). Esta teoría otorga una especial importancia a los distintos tipos de motivación, haciendo una distinción entre motivación autónoma, constituida por la motivación intrínseca y las regulaciones integrada e identificada; motivación controlada constituida por las regulaciones introyectada y externa; y desmotivación. Según la TAD, la motivación intrínseca refleja el compromiso de un sujeto con una actividad por el placer que le produce, sin necesidad de incentivos externos, y es la forma más deseable y duradera de motivación, y se relaciona con diversas variables comportamentales adaptativas (e.g., Zhang et al., 2011). Esta forma de regulación ha sido ampliamente estudiada y existe evidencia de su capacidad para identificar, cuando se presentan niveles bajos de la misma, patrones comportamentales menos adaptativos con independencia del nivel de otras regulaciones motivacionales (Hein et al., 2004; Zhang et al., 2011). Los resultados de una revisión sistemática llevada a cabo recientemente, en la que se han considerado 256 estudios que han abordado la motivación en EF desde la TAD, refuerzan estos hallazgos (Vasconcellos et al., 2019). El metaanálisis realizado ha puesto de manifiesto que la motivación autónoma se relaciona positivamente con variables adaptivas y negativamente con aquellas desadaptativas. La TAD postula que existen ciertas necesidades psicológicas, primarias y universales, que motivan el comportamiento humano: la autonomía, la competencia y la relación con los demás. Si bien durante los últimos años han emergido nuevas candidatas como la novedad o la moralidad, no se ha podido confirmar que dichas necesidades cumplan los criterios necesarios para ser consideradas primarias y universales (Vansteenkiste et al., 2020). Un alumno se siente autónomo cuando puede decidir determinados aspectos de su proceso de enseñanza-aprendizaje, normalmente porque el docente cede una mayor responsabilidad, favoreciendo sus intereses y preferencias (Reeve et al., 2014). Los estudiantes competentes se sienten capaces y con éxito en las tareas desarrolladas durante los procesos de aprendizaje. La necesidad de relación con los demás sugiere que los alumnos precisan sentir una conexión con su profesor, sus compañeros y su centro educativo. Cada una de estas tres necesidades juega un papel importante para el desarrollo y la experiencia óptima, y la satisfacción de estas influirá positivamente sobre la motivación intrínseca (Vansteenkiste et al., 2020). Este postulado de la TAD ha sido corroborado por numerosos estudios llevados a cabo en contextos de EF que han mostrado con unanimidad relaciones significativas de la satisfacción de autonomía (García-González et al., 2015) y de competencia (Jaakkola et al., 2016) con la motivación intrínseca, apareciendo con menor frecuencia la asociación entre la satisfacción de relación con los demás y la motivación intrínseca (e.g., Liu \& Chung, 2016; van Aart et al., 2017). Los resultados del metaanálisis realizado por Vasconcellos et al. (2019) pusieron de manifiesto que los distintos tipos de regulaciones motivacionales se asocian sistemáticamente con el apoyo a las NPB que muestran los profesores de EF.

Como muestra este metaanálisis, la literatura es prolija en estudios llevados a cabo en EF que eviden- 


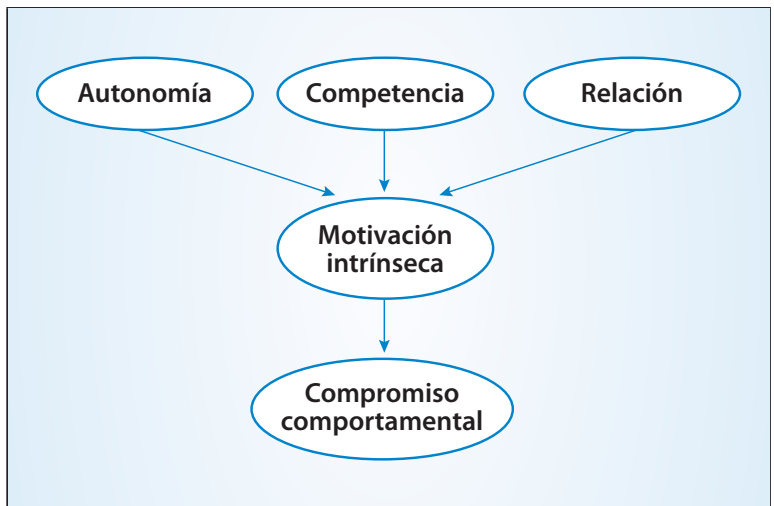

Figura 1. Modelo predictivo del compromiso comportamental testado.

cian la asociación de la motivación intrínseca con patrones motivacionales y conductuales considerados adaptativos (e.g., Jiménez Castuera et al., 2015). Los hallazgos de algunos de estos trabajos que han considerado el compromiso comportamental como variable objeto de estudio han corroborado que los alumnos que presentan una motivación autónoma, frente a una motivación controlada, tienden a mostrar mayor compromiso comportamental en la clase (e.g., Curran \& Standage, 2017; Yoo, 2015).

Los trabajos que han abordado el estudio del compromiso en EF han considerado al grupo de estudiantes como un todo, no contemplando posibles diferencias en función del nivel de práctica de AF (e.g., Van den Berghe et al., 2016). Sin embargo, hay evidencia de la existencia de perfiles motivacionales en clase de EF que difieren en cuanto a la práctica de AF que realizan en su tiempo libre (e.g., Franco et al., 2017). La distinta caracterización motivacional de los adolescentes en función de su adherencia a la práctica de AF sugiere la posibilidad de que la secuencia motivacional propuesta por la TAD en la explicación de la práctica de AF varíe en función de estos distintos perfiles. Existe evidencia, por ejemplo, de que la competencia percibida podría mediar la relación entre motivación intrínseca y práctica de $\mathrm{AF}$ en chicos, pero no en chicas (Erdvik et al., 2014). En este sentido, sería interesante comprobar si el nivel de AF podría ser un factor moderador en las relaciones establecidas entre distintas variables motivacionales y comportamentales.

Considerando lo anterior, el objetivo principal es profundizar en la comprensión de las diferencias en los patrones motivacionales que puedan explicar el compromiso comportamental en EF en función del nivel de AF que realicen los alumnos. Para ello, en un primer momento se analizan las diferencias entre estudiantes en la satisfacción de sus necesidades psicológicas básicas, su motivación intrínseca y su compromiso comportamental en función de su nivel de AF. En un segundo momento, se testa la validez de un modelo predictivo del compromiso comportamental a partir de la TAD (Figura 1) y, por último, se comprueba la invarianza de dicho modelo en grupos de estudiantes con diferente nivel de práctica de AF. Se hipotetiza, en primer lugar, que los participantes con un nivel alto de AF mostrarán puntuaciones más altas en la satisfacción de las necesidades psicológicas básicas, la motivación intrínseca y el compromiso comportamental. La segunda hipótesis del estudio es que todas las relaciones establecidas en el modelo que aparece en la Figura 1 serán significativas en sentido positivo. Por último, se hipotetiza que la asociación entre las variables de estudio diferirá en función del de práctica de AF debido a la existencia de diversos perfiles motivacionales entre los alumnos de EF. La ausencia de literatura al respecto nos impide formular en qué sentido variarán las relaciones entre las variables.

\section{Método}

\section{Participantes}

La muestra estuvo compuesta por 465 estudiantes (241 de género femenino) con edades comprendidas entre 12 y 17 años $(M=14.22$; $\mathrm{DE}=1.34)$ pertenecientes a los cursos de $1^{\circ}$ de educación secundaria obligatoria (ESO) $(\mathrm{n}=96), 2^{\circ}$ de ESO $(\mathrm{n}=115), 3^{\circ}$ de ESO ( $\mathrm{n}$ = 153), $4^{\circ}$ de ESO $(n=78)$ y $1^{\circ}$ de Bachillerato $(n=23)$. Se hizo un muestreo no probabilístico por conveniencia. Los sujetos pertenecían a 21 centros de educación secundaria de la Comunidad de Madrid, y recibían dos sesiones semanales de EF obligatoria con una duración de entre 50 minutos y una hora por sesión.

\section{Instrumentos}

Satisfacción de las Necesidades Psicológicas Básicas. Se utilizó la adaptación al castellano (Moreno et al., 2008) de la Escala de Medición de las Necesidades Psicológicas Básicas (BPNES; Vlachopoulos \& Michailidou, 2006). Este instrumento tiene como frase introductoria "En mis clases de EF..." y se compone de doce. Cuatro de ellos miden la satisfacción de autonomía (e.g., "los ejercicios que realizo se ajustan a mis intereses), cuatro, la satisfacción de competencia (e.g., "realizo los ejercicios eficazmente") y los otros cuatro, la satisfacción de relación con los demás (e.g., "me siento muy cómodo/a con mis compañeros/as"). En este estudio, la fiabilidad fue $\alpha=.75$ para la autonomía, $\alpha=.71$ para la competencia y $\alpha=.83$ para la relación con los demás. 
Motivación Intrínseca. Se utilizaron los cuatro ítems correspondientes al factor motivación intrínseca de la adaptación al castellano (Moreno et al., 2009) de la Escala del Locus Percibido de Causalidad (PLOC; Goudas et al., 1994). Este cuestionario se introduce con la frase "Participo en esta clase de EF..."; un ejemplo de ítem que compone la subescala de motivación intrínseca es "porque la EF es divertida". La fiabilidad en este estudio fue de $\alpha=.84$.

Compromiso en EF. Se utilizó el Cuestionario de Compromiso en EF (Shen et al., 2012). Este instrumento se introduce con el encabezado "En clase de EF" y consta de cinco ítems agrupados en un solo factor (e.g., "trabajo tanto como puedo"). La estructura factorial presentó índices de ajuste adecuados $(\chi 2[5]=79.08, \mathrm{p}$ $<.001, \mathrm{CFI}=.93, \mathrm{TLI}=.91, \mathrm{SRMR}=.06 \mathrm{y}$ una fiabilidad de $\alpha=.86$ en este trabajo.

Los instrumentos anteriores se contestaron mediante una escala Likert de 1 (totalmente en desacuerdo) a 5 (totalmente de acuerdo).

Nivel de práctica de AF. Se utilizó un ítem creado ad hoc ("En caso de que realices actividad físico-deportiva fuera de clase, ¿cuánto dirías que es tu nivel de práctica?") con las opciones de respuesta "poco", "normal", "bastante", "mucho". En el apartado de procedimiento se describe cómo se llevó a cabo el abordaje de esta pregunta durante la administración de los cuestionarios por parte del equipo investigador.

\section{Procedimiento}

Se estableció contacto con los equipos directivos y/o responsables del departamento de EF de los diferentes centros de enseñanza para informarles de los objetivos del estudio y pedirles su colaboración. Una vez obtenidas las autorizaciones pertinentes, los cuestionarios fueron administrados durante la clase de EF por un miembro del equipo investigador. Esta persona proporcionó una breve explicación del objetivo del estudio e informó a los participantes sobre la forma de completar el cuestionario. En relación con la pregunta de nivel de práctica de AF se les facilitaron ejemplos de cada una de las opciones y se respondió de forma individual a los alumnos que tenían dudas sobre su propio nivel, utilizando como referencia las recomendaciones de la Organización Mundial de la Salud para establecer los niveles "bastante" y "mucho" (World Health Organization, 2010). De este modo, se sugirió a los alumnos que solo realizaran $\mathrm{AF}$ como medio de transporte que marcaran nivel "bajo"; a los que realizaran AF moderada sin alcanzar los 60 minutos diarios, que marcaran "normal"; a los que alcanzaran 60 minutos diarios, que marcaran "bastante" y a los que hicieran AF todos los días y, al menos tres, esta fuera de intensidad alta, que marcaran "mucho". Se insistió en el anonimato de las respuestas solicitando sinceridad a los participantes. El tiempo requerido para completar el cuestionario fue de aproximadamente 20 minutos, variando ligeramente según la edad de los estudiantes. La recolección de datos siguió las indicaciones éticas de la Asociación de Psicología Americana (2002) y contó con la aprobación del Comité de Ética de una universidad pública de Madrid.

\section{Análisis de datos}

En un primer momento se calcularon los estadísticos descriptivos. Para determinar los grupos de estudio en función de la práctica de AF se segmentó la muestra considerándose el grupo de AF baja ( $n=240)$ aquel formado por los adolescentes que habían marcado poco o normal en el nivel de AF, y grupo de AF alta ( $n=225$ ) el constituido por adolescentes que habían indicado hacer bastante o mucha AF. A continuación, se llevó a cabo una prueba t para muestras independientes para analizar las diferencias entre el grupo de AF baja y AF alta.

En un segundo momento se testó un modelo de ecuaciones estructurales predictivo del compromiso en los adolescentes (Figura 2) a través del método de máxima verosimilitud. Con el objetivo de comprobar la validez del modelo, se consideraron diferentes índices de bondad de ajuste: ratio entre chi-cuadrado y grados de libertad ( $\chi 2 /$ g.1.), RMSEA (Root Mean Square Error of Approximation), SRMR (Standardized Root Mean Square Residual) y los índices incrementales CFI (Comparative Fit Index) y TLI (Tucker-Lewis Index). Estos índices de bondad de ajuste son considerados aceptables cuando $\chi 2 / g .1$. presenta valores entre 1 y 3 (Kline, 2005). Según Hu and Bentler (1999) es recomendable que el RMSEA muestre valores cercanos a 0.6, el SRMR muestre valores cercanos a 0.8 y los índices incrementales (CFI y TLI) estén cercanos a 0.95

Por último, se realizó un análisis multigrupo para testar si el modelo resultante era estable en función del nivel de práctica de AF de los participantes. Para llevar a cabo este análisis multigrupo se siguieron las indicaciones de Byrne (2001). En la Tabla 2 se presentan los índices de ajuste de los tres modelos sometidos a prueba. Según las recomendaciones de Cheung y Rensvold (2002), una diferencia entre los CFI ( $\Delta C F I)$ menor o igual a 0.01 entre dos modelos con diferentes restricciones indica una disminución no sustancial de ajuste del modelo. Se utilizaron los paquetes estadísticos SPSS y AMOS en su versión 24 para el análisis de los datos. 
Tabla 1. Estadísticos descriptivos de las variables de estudio en función del nivel de práctica de AF.

\begin{tabular}{|c|c|c|c|}
\hline & $\begin{array}{l}\text { Muestra total } \\
\quad(n=465)\end{array}$ & $\begin{array}{l}\text { Nivel AF Bajo } \\
\quad(n=240)\end{array}$ & $\begin{array}{l}\text { Nivel AF Alto } \\
\quad(n=225)\end{array}$ \\
\hline Autonomía & $3.22(.91)$ & $3.18(1.00)$ & $3.29(.79)$ \\
\hline Competencia & $3.89(.61)$ & $3.60(.75)$ & $4.15(.59)$ ** \\
\hline Relación con los demás & $4.20(.78)$ & $4.11(.86)$ & $4.31(.69) * *$ \\
\hline Motivación intrínseca & $3.93(.81)$ & $3.69(.89)$ & $4.18(.73) * *$ \\
\hline Compromiso comportamental & $4.18(.73)$ & $4.16(.69)$ & $4.21(.78)$ \\
\hline
\end{tabular}

$* * \mathrm{p}<.05$

Tabla 2. Análisis de invarianza multigrupo en función del nivel de autodeterminación y de AF.

\begin{tabular}{|c|c|c|c|c|c|c|}
\hline Restricciones & $X^{2}$ (g.l.) & p & $\mathrm{CFI}$ & RMSEA & SRMR & $\Delta \mathrm{CFI}$ \\
\hline Invarianza configural & $424.17(196)$ & .001 & .919 & .05 & .06 & --- \\
\hline Invarianza métrica & $451.29(207)$ & .001 & .915 & .05 & .06 & .004 \\
\hline Invarianza estructural & $628.78(226)$ & .001 & .858 & .06 & .07 & .057 \\
\hline
\end{tabular}

\section{Resultados}

Estadísticos descriptivos y diferencias en función del nivel de AF

La Tabla 1 muestra los estadísticos descriptivos de las variables de estudio en función de los grupos creados según el nivel de AF (bajo vs. alto).

La prueba $t$ para muestras independientes reveló que el grupo de alta AF mostró puntuaciones más elevadas en todas las variables de estudio, siendo dicha diferencia significativa en la satisfacción de competencia $(\mathrm{t}=-9.01, p=.001)$, relación con los demás $(\mathrm{t}=-2.77, p=$ .006), y motivación intrínseca $(\mathrm{t}=-6.52, p=.001)$, pero no así en la satisfacción de autonomía ( $\mathrm{t}=-1.23, p=.22)$, y en el compromiso comportamental $(t=-.86, p=.39)$ en función del nivel de AF.

\section{Análisis de ecuaciones estructurales e invarianza en función del nivel de AF}

En un primer momento se testó el modelo de medición considerando como indicadores cada uno de los ítems que componían los instrumentos para medir las distintas variables de estudio. En línea con lo apuntado en trabajos anteriores (Barclay et al., 1995; Chin, 1998) en el presente estudio se decidió usar el criterio de aceptación de carga factorial de 0.50 . Con este criterio se eliminaron del modelo de medición el ítem 4 de relación con los demás, el ítem 1 de competencia, el ítem 1 de motivación intrínseca y los ítems 1 y 3 de compromiso debido a que la carga factorial en su variable latente correspondiente fue inferior a 0.50 .

El modelo de ecuaciones estructurales llevado a cabo indicó que la asociación entre satisfacción de la nece-

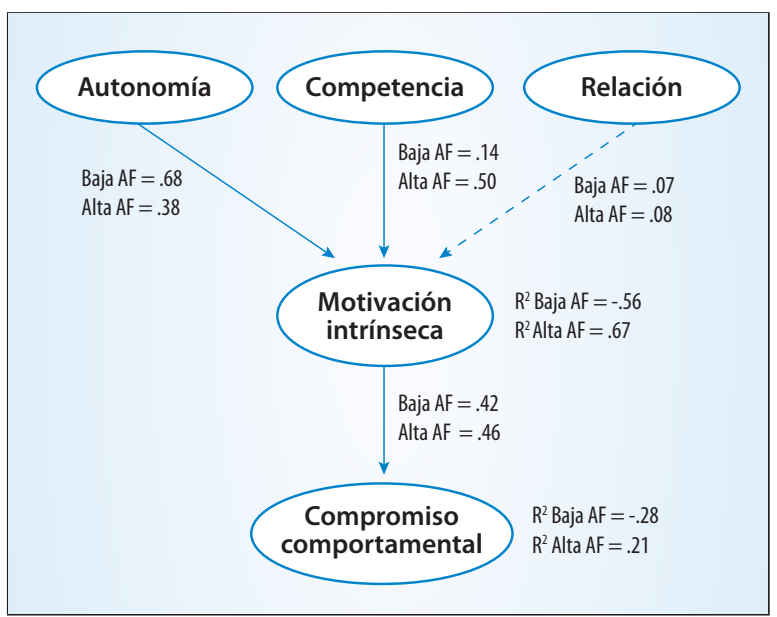

Figura 2. Modelo explicativo del compromiso. La línea discontinua representa una relación que se hipotetizó pero que no resultó significativa por lo que la relación con los demás queda excluida del modelo resultante. Se muestran las $\beta$ de esta asociación en el modelo antes de suprimir la asociación entre relación con los demás y motivación intrínseca.

sidad de relación y la motivación intrínseca no resultó significativa $(\beta=-0.01 ; \mathrm{p}=.91)$ y se decidió quitar esta relación de la secuencia hipotetizada. El modelo resultante (Figura 2) mostró unos adecuados índices de bondad de ajuste $(\chi 2[112]=310.65, \mathrm{p}<.001, \chi 2 / \mathrm{df}=2.77, \mathrm{CFI}=$ $.94, \mathrm{TLI}=.92, \mathrm{RMSEA}=.06, \mathrm{SRMR}=.06)$ encontrándose una relación significativa y positiva de la satisfacción de autonomía con la motivación intrínseca $(\beta=0.55$; $p$ $<$.001); de la satisfacción de competencia con la motivación intrínseca $(\beta=0.30 ; p<.001)$; y de la motivación intrínseca con el compromiso $(\beta=0.44 ; \mathrm{p}<.001)$.

En la Tabla 2 se presentan los índices de ajuste de los tres modelos sometidos a prueba. Se observa la existencia de una invarianza configural, en tanto que el modelo sin restricciones muestra unos índices 
de ajuste adecuados. Esto significa que el número de factores y su asociación con los diferentes indicadores es similar en ambos grupos. En un segundo modelo anidado el $\triangle C F I<.01$ sugiere la invarianza métrica en ambos grupos. Esto es, las cargas factoriales pueden ser consideradas equivalentes en ambos grupos. Sin embargo, la disminución de .057 en el CFI al añadir la restricción de los pesos de regresión y covarianzas indican la ausencia de invarianza estructural (esto es, la asociación entre las variables latentes no se considera equivalente en ambos grupos). Al comparar los pesos de regresión estandarizados en el modelo de invarianza métrica (con restricción en las cargas factoriales) se observa que la asociación entre competencia y motivación intrínseca no resulta significativa en el grupo de baja $\mathrm{AF}(\beta=.14 ; p=.086)$ y el peso de regresión estandarizado de la variable autonomía sobre la motivación intrínseca es más elevado en el grupo de baja $\mathrm{AF}(\beta=.68 ; p<.001)$ que en el grupo de AF alta. $(\beta=.38 ; p=.001)$. El $r^{2}$ ajustado de la variable compromiso en el modelo propuesto también mostró unos índices similares en el grupo de baja (28\% de varianza explicada) y alta AF (21\% de varianza explicada).

\section{Discusión}

El objetivo principal de este trabajo fue profundizar en la comprensión de las diferencias en los patrones motivacionales que puedan explicar el compromiso comportamental en EF en función del nivel de AF que realicen los alumnos.

Para ello, en un primer momento se analizaron las diferencias entre estudiantes en la satisfacción de sus necesidades psicológicas básicas, su motivación intrínseca y su compromiso comportamental en función de su nivel de AF. Los resultados pusieron de manifiesto que los alumnos con un nivel alto de práctica de AF mostraron un patrón motivacional más adaptativo en la clase de EF, con puntuaciones más elevadas en la satisfacción de competencia, relación con los demás y motivación intrínseca que el grupo de estudiantes de nivel de AF bajo.

Estos hallazgos están en línea con trabajos anteriores que habían sugerido la validez de los postulados de la TAD en el contexto de EF en la predicción de la práctica de actividad física. Jaakkola et al. (2016) encontraron asociación entre la práctica de $\mathrm{AF}$ extraescolar y una mayor competencia motriz. Se podría pensar que los alumnos con alto nivel de AF se sientan más competentes en clase de EF ya que se enfrentan a situaciones para las que se perciben más preparados debido a sus experiencias extraescolares. Como han sugerido estudios previos que han encontrado una asociación entre variables psicosociales y de rendimiento físico, probablemente este aumento de competencia se deba a la mejora de la condición física (e.g., Dunton et al., 2007). Por otro lado, existe evidencia de la relación entre la satisfacción de relación con los demás y la AF realizada por adolescentes medida objetivamente (Brunet et al., 2016). En cuanto a la asociación entre motivación intrínseca y práctica de $\mathrm{AF}$, la literatura es prolija en estudios que han puesto de manifiesto la influencia que la experiencia de este tipo de regulación tiene sobre la práctica de AF (e.g., Franco et al., 2017).

En el presente estudio no se encontraron diferencias en la satisfacción de autonomía y el compromiso comportamental en EF en función del nivel de AF. Estudios anteriores han sugerido que la satisfacción de autonomía en clase de EF se asocia con el nivel de AF fuera de ella (Standage et al., 2003). Sin embargo, también se ha encontrado que la fuerza de esta asociación es menor que la existente entre las otras NPB y la práctica de AF (Cox et al., 2008). En esta línea, Carrasco et al. (2015), pusieron en marcha un programa extraescolar de juegos reducidos en fútbol y valorar el impacto de este sobre la satisfacción de las NPB y la motivación en EF. Al comparar al grupo experimental con el grupo control (que no había participado en ningún programa extraescolar) no se hallaron diferencias significativas en la satisfacción de autonomía. Por último, llama la atención la ausencia de diferencias significativas en el compromiso comportamental en clase de EF en función de la AF. Si bien existe evidencia de la relación entre algunos elementos del compromiso emocional como la diversión y los niveles de AF en contextos deportivos (e.g., Ullrich-French \& Smith, 2009), no se han encontrado en la literatura estudios que hayan analizado la asociación entre compromiso (emocional, comportamental o cognitivo), y nivel de AF.

Los hallazgos relacionados con este primer objetivo coinciden en su mayor parte con la literatura existente. En los aspectos en los que los resultados de este estudio no están en línea con la literatura general se abren sugerentes interrogantes. Por un lado, comprender por qué la necesidad de autonomía en el aula de EF no tiene una relación tan fuerte como las otras dos necesidades con el nivel de AF, como evidencian los pocos estudios encontrados. Y por otro, la necesidad de abrir una línea de investigación que indague en la asociación entre el compromiso comportamental en clase de EF y la práctica de AF.

En un segundo momento, se testó la validez de un modelo predictivo del compromiso comportamental a partir de la TAD (Figura 1). De acuerdo a los postulados teóricos de la mencionada teoría se hipotetizó que 
las tres necesidades psicológicas básicas predecirían la motivación intrínseca (Ryan \& Deci, 2020).

Como se hipotetizó, la satisfacción de autonomía y de competencia se asoció positivamente con la motivación intrínseca. Sin embargo, la asociación entre la necesidad de relación y la motivación intrínseca no resultó ser significativa y fue, por tanto, eliminada del modelo definitivo.

Estudios anteriores desarrollados en el contexto de EF bajo el marco de la TAD son coincidentes en que tanto la autonomía como la competencia percibidas en clase de EF emergen con más frecuencia y/o más fuerza como predictoras de la motivación intrínseca tanto en la misma clase de EF (Standage et al., 2012) como en contextos de ejercicio físico (Liu \& Chung, 2016).

En el reciente metaanálisis elaborado por Vasconcellos et al. (2019) se muestra también la existencia de una asociación entre la necesidad de relación y motivación autónoma, aunque con menor fuerza. Otros trabajos han coincidido en sugerir este rol secundario de la satisfacción de la relación con los demás al tratar de explicar otros constructos adaptivos. En esta línea, se ha encontrado que la satisfacción de autonomía y de competencia resultan influyentes en la experiencia del flow disposicional y la adopción de hábitos saludables (Ferriz et al., 2016); o que la satisfacción de competencia se asocia con las calificaciones del alumnado (Sevil et al., 2017), no habiéndose detectado una asociación entre estas variables y la satisfacción de relación con los demás. Los hallazgos de la presente investigación, en línea con los estudios anteriormente mencionados, sugieren que, si bien el papel de la relación con los demás es un factor relevante en la explicación de diversas variables adaptativas relacionadas con la práctica de $\mathrm{AF}$ desde el prisma de la TAD, su relación con un interés intrínseco hacia las actividades realizadas en EF parece no estar tan clara como lo está la relación de dicho interés con la satisfacción de las necesidades de autonomía y competencia. Vasconcellos et al. (2019) han sugerido que, mientras que el papel del docente parece propiciar experiencias motivacionales a través de la percepción de competencia y autonomía, la satisfacción de relación con los demás se ve más influida por la relación con los iguales. En línea con esta idea, los hallazgos de nuestro estudio podrían sugerir que el rol del profesor de EF es más importante que el de los compañeros a la hora de favorecer la motivación intrínseca en este contexto. Recientemente, se ha sugerido que la satisfacción de relación con los demás podría actuar como un antecedente de la satisfacción de la autonomía y la competencia (Holt et al., 2018). Sería interesante que futuros estudios indagaran en el comportamiento de esta variable en el contexto de EF.
De acuerdo a todo lo anterior, sería recomendable que los profesores de EF fomentaran el apoyo a las NPB a través de diversas estrategias que han resultado efectivas en estudios previos (Standage \& Ryan, 2012). Por ejemplo, el otorgar a los alumnos un papel importante en la toma de decisiones o proporcionar explicaciones racionales a las actividades y/o normas que se dan en clase, favorecerá la satisfacción de autonomía. Por otro lado, los profesores pueden fomentar la satisfacción de competencia de sus alumnos proporcionando una información clara con respecto a la estructura de la clase y los objetivos perseguidos, diseñando actividades que a los alumnos les supongan un desafío óptimo o aportando abundante feedback positivo. Por último, para favorecer la necesidad de relación, es fundamental que los profesores se muestren empáticos e interesados por sus alumnos. Además, teniendo en cuenta el papel fundamental que los compañeros tienen en la satisfacción de esta necesidad, sería interesante poner en práctica estilos de enseñanza participativos que permitieran a los alumnos percibir a sus compañeros como figuras relevantes en su proceso de aprendizaje.

En una segunda parte del modelo se hipotetizó que la motivación intrínseca en el aula de EF se relacionaría con el compromiso comportamental en la misma. Los resultados del presente trabajo están en línea con el grueso de estudios llevados a cabo anteriormente en contextos de EF entre adolescentes que han probado la asociación positiva entre motivación intrínseca y el compromiso de los alumnos (e.g., Cuevas et al., 2018). La TAD sugiere que es más probable que los alumnos se involucren en actividades de aprendizaje por el disfrute y la satisfacción inherentes a la actividad que por recompensas externas como las calificaciones o el refuerzo positivo del alumno, a pesar de que estos reforzadores externos también pueden afectar al desarrollo de conductas (Ryan \& Deci, 2020). Un estudio reciente ha sugerido que cuando los profesores de EF crean un ambiente estructurado y proporcionan apoyo a la autonomía los alumnos mejoran su compromiso en clase (Cheon et al., 2020). Serían necesarias investigaciones en esta línea para mejorar la comprensión de los antecedentes que expliquen el compromiso de los alumnos en clase de EF.

Por último, se comprobó la invarianza del modelo resultante en grupos de estudiantes con diferente nivel de práctica de AF.

Los resultados del análisis revelaron que la relación entre la satisfacción de la necesidad de competencia y la motivación intrínseca no resultó significativa en los alumnos del grupo de nivel bajo, alcanzando la satisfacción de la necesidad de autonomía un mayor poder predictivo de dicha motivación. En el caso de los estudiantes del grupo de nivel alto de AF, tanto la satis- 
facción de autonomía como la satisfacción de competencia resultaron significativas, si bien en este caso fue la satisfacción de competencia la que adquirió mayor relevancia en la predicción de la motivación intrínseca.

No se han encontrado estudios que hayan testado la invarianza de un modelo similar en función de la AF de los participantes. Sin embargo, existen estudios previos que sugieren que la percepción de competencia puede ser especialmente importante en la predicción de comportamientos adaptativos entre aquellos sujetos más activos. Erdvik et al. (2014) encontraron que el rol de la percepción de competencia como mediador entre el índice de autodeterminación y la intención de ser físicamente activo resultaba significativo en chicos (habitualmente más activos), pero no en chicas (Meneses \& Ruiz Juan, 2017). Del mismo modo, se ha sugerido que la satisfacción de autonomía adquiere un papel especialmente relevante entre adolescentes con patrones poco adaptativos. En un estudio cualitativo llevado a cabo con estudiantes que mostraban bajos niveles de compromiso en clase de EF, Mitchell et al. (2015) concluyeron que, si bien la satisfacción de las tres NPB era importante, la percepción de autonomía suponía una condición necesaria para el desarrollo de la motivación intrínseca.

Lo señalado anteriormente sugiere que la satisfacción de autonomía podría cobrar una mayor relevancia en los patrones menos adaptativos, y la satisfacción de competencia una importancia mayor en los patrones de comportamiento más adaptativos.

Considerando los hallazgos de Van den Berghe et al. (2016), la comprensión de los procesos subyacentes al compromiso comportamental adquiere en la actualidad una especial relevancia. En dicho trabajo se detecta que la relación entre la motivación de los profesores y el compromiso de los alumnos es bidireccional, de modo que el compromiso que los estudiantes muestran en clase de EF va a influir en la satisfacción y frustración de las NPB de los profesores, lo que acarreará determinadas consecuencias en el proceso de enseñanza-aprendizaje.

Este trabajo pretende aportar sugerencias para comprender la relación entre el nivel de práctica de AF y las variables que afectan al comportamiento del alumnado. Las aportaciones de las investigaciones de los últimos años nos sugieren una clara relación entre el nivel de $\mathrm{AF}$ y patrones adaptativos y comportamentales más positivos. Sin embargo, para comprender en profundidad las razones de esta realidad, sería necesario ampliar el marco de referencia, intentando aportar luz sobre las razones que llevan al alumnado a practicar menos AF. En este sentido, diversos trabajos que han estudiado esta relación bajo un enfoque cualitativo han detectado que, en gran medida, la baja práctica de AF puede ser una consecuencia de las experiencias negativas vividas, concretamente en las clases de EF. Aspectos como una violencia simbólica expresada por comportamientos de rechazo, exclusión o devaluación (Beltrán-Carrillo et al., 2012) o un modelo docente excesivamente centrado en la promoción de la salud o en la valoración del rendimiento físico desde un discurso de masculinidad hegemónica (Beltrán-Carrillo \& Devís-Devís, 2019). Sería interesante la realización de investigaciones con métodos mixtos para establecer una visión más completa de las relaciones de causalidad entre variables emergentes.

Este estudio posee algunas limitaciones que conviene señalar. Por un lado, ante la necesidad de acortar el cuestionario de recogida de información, la medición de la AF se ha realizado mediante un cuestionario subjetivo, lo que puede conllevar cierto grado de error en su medida (Sirard \& Pate, 2001). Esta importante limitación fue tenida en cuenta durante la administración de los cuestionarios, tal y como se describe en el apartado de procedimiento. Por otro lado, se trata de un estudio transversal del que no se pueden inferir relaciones causales.

Sería interesante que futuros estudios profundizasen en el análisis del rol de la necesidad de relación para alcanzar una mejor comprensión de esta variable en el marco de la TAD. Por otra parte, parece necesario utilizar en futuros trabajos un enfoque centrado en la persona para avanzar en el conocimiento del comportamiento de las variables motivacionales y de compromiso en sujetos con distintas características con el fin de último de diseñar estrategias adaptadas a los distintos perfiles, prestando una especial atención a aquellos alumnos menos motivados quienes habitualmente se asocian con patrones comportamentales menos adaptativos.

\section{Conclusiones}

El presente trabajo se suma a la literatura existente relativa al análisis de variables motivacionales con el compromiso mostrado en clases de EF. Los hallazgos apuntan, en línea con trabajos anteriores, a que el rol de la satisfacción de la necesidad de relación no resulta tan determinante como el de la necesidad de autonomía y de competencia en la explicación de la motivación intrínseca. Los resultados revelan que el modelo propuesto resulta adecuado para predecir el compromiso comportamental, si bien la ausencia de invarianza estructural en el modelo predictivo testado, sugiere que la secuencia motivacional que subyace al compromiso comportamental en EF difiere según el nivel de práctica de $\mathrm{AF}$ de los adolescentes. 
Albarracín, A., Moreno-Murcia, J. A., \& Beltrán-Carrillo, V. J. (2014). La situación actual de la educación física según su profesorado: Un estudio cualitativo con profesores de la Región de Murcia. Cultura, Ciencia y Deporte, 9(27), 225-234.

American Psychological Association. (2002). Ethical principles of psychologists and code of conduct (Amended August 3, 2016). American Psychological Association.

Barclay, D., Higgins, C., \& Thompson, R. (1995). The Partial Least Squares (PLS) approach to causal modelling: Personal computer adoption and use as an illustration. Technology Studies, 2(Special Issue on Research Methodology, 2), 285-309.

Beltrán-Carrillo, V. J., \& Devís-Devís, J. (2019). El pensamiento del alumnado inactivo sobre sus experiencias negativas en educación física. Ricyde, 15 (extra 55), 20-34. doi:10.5232/ricyde2019.05502

Beltrán-Carrillo, V. J., Devís-Devís, J., Peiró, C., \& Brown, D. (2012). When Physical Activity Participation Promotes Inactivity: Negative Experiences of Spanish Adolescents in Physical Education and Sport. Youth and Society, 44(1), 3-27.

Brunet, J., Gunnell, K. E., Teixeira, P. J., Sabiston, C. M., \& Bélanger, M. (2016). Should we be looking at the forest or the trees? Overall psychological need satisfaction and individual needs as predictors of physical activity. Journal of Sport \& Exercise Psychology, 38, 317-330. doi:10.1123/jsep.2016-0256

Byrne, B. M. (2001). Structural equation modeling with Amos: Basic concepts, applications, and programming. Erlbaum.

Carrasco, H., Chirosa, L. J., Martín, I., Cajas, B., \& Relgal, R. E. (2015). Efectos de un programa extraescolar basado en juegos reducidos sobre la motivación y las necesidades psicológicas básicas en las clases de educación física. Revista Iberoamericana de Psicología del Ejercicio y el Deporte, 10(1), 23-31.

Cox, A., Smith, A., \& Williams, L. (2008). Change in physical education motivation and physical activity behaviour during middle school. Journal of Adolescent Health, 43, 506 - 513. doi:10.1016/j. jadohealth.2008.04.020

Cuevas, R., García-Calvo, T., González, J., \& Fernández-Bustos, J. G. (2018). Necesidades psicológicas básicas, motivación y compromiso en educación física. Revista de Psicología del Deporte, 27(1), 97-104.

Curran, T., \& Standage, M. (2017). Psychological needs and the quality of student engagement in Physical Education: teachers as key facilitators. Journal of Teaching in Physical Education, 36, 262-276. doi:10.1123/jtpe.2017-0065

Cheon, S. H., Reeve, J., \& Vansteenkiste, M. (2020). When teachers learn how to provide classroom structure in an autonomy-supportive way: Benefits to teachers and their students. Teaching and Teacher Education, 90, 103004. doi:10.1016/j.tate.2019.103004

Cheung, G. W., \& Rensvold, R. B. (2002). Evaluating goodness-of-fit indexes for testing measurement invariance. Structural Equation Modeling, 9(2), 233-255. doi:10.1207/S15328007SEM0902_5

Chin, W. W. (1998). Issues and opinion on structural equation modeling. MIS Quarterly, 22(1), 7-17.

Dunton, G. F., Schneider, M., \& Cooper, D. M. (2007). An investigation of psychosocial factors related to changes in physical activity and fitness among female adolescents. Psychology \& Health, 22(8), 929-944. doi:10.1080/14768320601124915

Erdvik, I. B., Overby, N. C., \& Haugen, T. (2014). Students' self-determined motivation in physical education and intention to be physically active after graduation: The role of perceived competence and identity. Journal of Physical Education and Sport, 14(2), 232-241. doi:10.7752/jpes.2014.02035

Ferriz, R., González-Cutre, D., Sicilia, A., \& Hagger, M. (2016). Predicting healthy and unhealthy behaviors through physical education: A self-determination theory-based longitudinal approach. Scandinavian Journal of Medicine \& Science in Sports, 26, 579-592. doi:10.1111/ sms. 12470

Franco, E., Acha, A., Gómez, V., \& Ricetti, A. (2019). Compromiso y motivación del alumnado en función de las necesidades psicológicas básicas de sus profesores en educación física. Revista Española de Educación Física y Deportes, 426, 289-296.
Franco, E., Coterón, J., Martínez, H. A., \& Brito, J. (2017). Perfiles motivacionales en estudiantes de educación física de tres países y su relación con la actividad física. Suma Psicológica, 24(1), 1-8. doi:1016/j. sumpsi.2016.07.001

Fredricks, J. A., Blumenfeld, P. C., \& Paris, A. H. (2004). School engagement: potential of the concept, state of the evidence. Review of Educational Research, 74(1), 59-109. doi:10.3102/00346543074001059

García-González, L., Aibar, A., Sevil, J., Almolda-Tomás, F. J., \& Julián Clemente, J. A. (2015). Soporte de autonomía en Educación Física: evidencias para mejorar el proceso de enseñanza. Cultura, Ciencia y Deporte, 10, 103-111.

Goudas, M., Biddle, S., \& Fox, K. (1994). Perceived locus of causality, goal orientations, and perceived competence in school physical education classes. British Journal of Educational Psychology, 64, 453-463.

Hein, V., Müür, M., \& Koka, A. (2004). Intention to be physically active after school graduation and its relationship to three types of intrinsic motivation. European Physical Education Review, 10(1), 5-19. doi:10.1177/1356336X04040618

Holt, A. D., Smedegaard, S., Pawloski, C. S., Skovgaard, T., \& Christiansen, L. B. (2018). Pupils' experiences of autonomy, competence and relatedness in "Move for well-being in schools": A physical activity intervention. European Physical Education Review. doi:10.1177/ $1356336 \mathrm{X} 18758353$

Hospel, V., Galand, B., \& Janosz, M. (2016). Multidimensionality of behavioural engagement: Empirical support and implications. International Journal of Educational Research, 77, 37-49. doi:10.1016/j. ijer.2016.02.007

Hu, L., \& Bentler, P. M. (1999). Cutoff criteria for fit indexes in covariance structure analysis: Conventional criteria versus new alternatives. Structural Equation Modeling, 6(1), 1-55.

Jaakkola, T., Yli-Piipari, S., Anthony, W., \& Liukkonen, J. (2016). Perceived physical competence towards physical activity, and motivation and enjoyment in physical education as longitudinal predictors of adolescents' self-reported physical activity. Journal of Science and Medicine in Sport, 19(9), 750-754. doi:10.1016/j.jsams.2015.11.003

Jiménez Castuera, R., Moreno, B., Leyton, M., \& Claver, F. (2015). Motivación y estadios de cambio para el ejercicio físico en adolescentes. Revista Latinoamericana de Psicología, 47(3), 196-204.

Kline, R. B. (2005). Principles and practice of structural equation modeling, 2nd ed. Guilford Press.

Ladwig, M. A., Vazou, S., \& Ekkekakis, P. (2018). "My Best Memory Is When I Was Done with It": PE Memories Are Associated with Adult Sedentary Behavior. Translational Journal of the American College of Sports Medicine, 3(16).

Liu, J. D., \& Chung, P.-K. (2016). Students' perceived autonomy support and psychological needs satisfaction in physical education and exercise intrinsic motivation. Journal of Sport Behavior, 39(4), 409.

Meneses, M., \& Ruiz Juan, F. (2017). Estudio longitudinal de los comportamientos y el nivel de actividad físico-deportiva en el tiempo libre en estudiantes de Costa Rica, México y España. Retos, 31, 219-226.

Mitchell, F., Gray, S., \& Inchley, J. (2015). “This choice thing really works..." Changes in experiences and engagement of adolescent girls in physical education classes, during a school-based physical activity programme. Physical Education and Sport Pedagogy, 20(6), 593-611. do i:10.1080/17408989.2013.837433

Molina, P., Valenciano, J., \& Úbeda-Colomer, J. (2016). El diseño curricular de la Educación Física en España: Una revisión crítica desde la LOGSE a la LOMCE. Cultura, Ciencia y Deporte, 11(32), 97-106.

Moreno, J. A., González-Cutre, D., \& Chillón, M. (2009). Preliminary validation in Spanish of a scale designed to measure motivation in physical education classes: The perceived locus of causality (PLOC) scale. The Spanish Journal of Psychology, 12(1), 327-337. doi:10.1017/ S1138741600001724

Moreno, J. A., González-Cutre, D., Chillón, M., \& Parra, N. (2008). Adaptación a la educación física de la escala de las necesidades psicológicas básicas en el ejercicio. Revista Mexicana de Psicología, 25(2), 295-303.

Reeve, J., Vansteenkiste, M., Assor, A., Ahmad, I., Cheon, S. H., Jang, H., Kaplan, H., Moss, J. D., Olaussen, B. S., \& Wang, J. C. K. (2014). The 
beliefs that underlie autonomy-supportive and controlling teaching: A multinational investigation. Motivation and emotion, 38, 93-110.

Ryan, R. M., \& Deci, E. L. (2020). Intrinsic and extrinsic motivation from a self-determination theory perspective: Definitions, theory, practices, and future direction. Contemporary Educational Psychology. doi:10.1016/j.cedpsych.2020.101860

Sevil, J., Aibar, A., Abós, A., \& García-González, L. (2017). El clima motivacional del docente de Educación Física: ¿Puede afectar a las calificaciones del alumnado? Retos, 31, 94-97.

Shen, B., McCaughtry, N., Martin, J., Fahlman, M., \& Garn, A. (2012). Urban high-school girls' sense of relatedness and their engagement in Physical Education. Journal of Teaching in Physical Education, 31, 231-245

Sirard, J. R., \& Pate, R. R. (2001). Physical activity assessment in children and adolescents. Sports Medicine, 31(6), 439-454.

Skinner, E., Furrer, C., Marchand, G., \& Kinderman, T. (2008). Engagement and disaffection in the classroom: Part of a larger motivational dynamic? Journal of Educational Psychology, 100, 765-781. doi:10.1037/a0012840

Standage, M., Duda, J. L., \& Ntoumanis, N. (2003). A model of contextual motivation in physical education: Using constructs from self-deter mination and achievement goal theories to predict physical activity intentions. Journal of Educational Psychology, 95(1), 97 - 110.

Standage, M., Gillison, F., Ntoumanis, N., \& Treasure, D. C. (2012). Predicting students' physical activity and health-related well-being: A prospective cross-domain investigation of motivation across school physical education and exercise settings. Journal of Sport \& Exercise Psychology, 34, 37-60.

Standage, M., \& Ryan, R. M. (2012). Self-Determination theory and exercise motivation: Facilitating self-regulatory processes to support and maintain health and well-being. In G. Roberts \& D. C. Treasure (Eds.), Advances in motivation in sport and exercise (pp. 233-270). Human Kinetics.

Ullrich-French, S., \& Smith, A. (2009). Social and motivational predictors of continued youth sport participation. Psychology of Sport and Exercise, 10, 87-96. van Aart, I., Hartman, E., Elferink-Gemser, M., Mombarg, R., \& Visscher, C. (2017). Relations among basic psychological needs, PE-motivation and fundamental movement skills in 9-12-year-old boys and girls in Physical Education. Physical Education and Sport Pedagogy, 22(1), 1520. doi:10.1080/17408989.2015.1112776

Van den Berghe, L., Cardon, G., Tallir, I. B., Kirk, D., \& Haerens, L. (2016) Dynamics of need-supportive and need-thwarting teaching behavior: the bidirectional relationship with student engagement and disengagement in the beginning of a lesson. Physical Education and Sport Pedagogy, 21(6), 653-670. doi:10.1080/17408989.2015.1115008

Vansteenkiste, M., Ryan, R. M., \& Soenens, B. (2020). Basic psychological need theory: Advancements, critical themes, and future directions. Motivation and emotion, 44(1), 1-31. doi:10.1007/s11031-01909818-1

Vasconcellos, D., Parker, P. D., Hilland, T., Cinelli, R., Owen, K. B., Kapsal, N., Lee, J., Antczak, D., Ntoumanis, N., Ryan, R. M., \& Lonsdale, C. (2019). Self-determination theory applied to physical education: A systematic review and meta-analysis. Journal of Educational Psychology, No Pagination Specified-No Pagination Specified. doi:10.1037/ edu0000420

Vlachopoulos, S., \& Michailidou, S. (2006). Development and initial validation of a measure of autonomy, competence and relatedness: the Basic Psychological Needs in Exercise Scale. Measurement in Physical Education \& Exercise Science, 10, 179-201. doi:10.1207/s15327841mpee1003_4

World Health Organization. (2010). Global Recommendations on Physical Activity for Health. Geneva, Switzerland: WHO Press.

Yoo, J. (2015). Perceived autonomy support and behavioral engagement in physical education: A conditional process model of positive emotion and autonomous motivation. Perceptual \& Motor Skills: Exercise \& Sport, 120(3), 731-746. doi:10.2466/06.PMS.120v20x8

Zhang, T., Solmon, M. A., Kosma, M., Carson, R. L., \& Gu, X. (2011) Need Support, Need Satisfaction, Intrinsic Motivation, and Physical Activity Participation among Middle School Students. Journal of Teaching in Physical Education, 30(1), 51-68. 